\title{
Magnetic Protein Microspheres as Dynamic Contrast Agents for Magnetomotive Optical Coherence Tomography
}

\author{
Freddy T. Nguyen*a, d, e, f, g, Elizabeth M. Dibbern ${ }^{\mathrm{a}, \mathrm{g}}$, Eric J. Chaney ${ }^{\mathrm{d}, \mathrm{g}}$, Amy L. Oldenburg ${ }^{\mathrm{d}, \mathrm{g}}$, \\ Kenneth S. Suslick ${ }^{\mathrm{a}, \mathrm{d}, \mathrm{g}}$, Stephen A. Boppart, c, d, f, g, h \\ ${ }^{\mathrm{a}}$ Department of Chemistry; ${ }^{\mathrm{b}}$ Department of Electrical and Computer Engineering; ${ }^{\mathrm{c}}$ Department of \\ Bioengineering; ${ }^{\mathrm{d} B e c k m a n}$ Institute for Advanced Science and Technology; ${ }^{\mathrm{e}}$ Medical Scholars \\ Program; ${ }^{\mathrm{f}}$ College of Medicine; ${ }^{\mathrm{g}}$ University of Illinois at Urbana-Champaign, 405 North Mathews \\ Avenue, Urbana, IL, USA 61801; ${ }^{\text {h}}$ Mills Breast Cancer Institute, Carle Foundation Hospital, 611 \\ West Park Street, Urbana, IL, USA 61801
}

\begin{abstract}
Optical coherence tomography (OCT) is an emerging biomedical imaging modality that has been developed over the last 15 years. More recently, OCT has been used for the intraoperative imaging of tumor margins in breast cancer and axillary lymph nodes providing a real time in-vivo assessment of the tissue morphology. Traditional OCT images are limited by only being able to observe morphological structures. As diagnostic medicine continues to push for earlier detection, one must develop functional imaging modalities that would detect molecular information in-vivo allowing a real-time microscopic analysis of the tissue specimen. A novel modality of OCT called magnetomotive-OCT (MMOCT) has been developed by our group, employing an induced modulated magnetic field with a magnetic contrast agent to create the added contrast to structural OCT images. Modified protein microspheres with a BSA protein shell functionalized with RGD peptide sequences for targeting and an oil core have been designed and synthesized. Magnetic nanoparticles $\left(\mathrm{Fe}_{3} \mathrm{O}_{4}\right)$ and Nile Red dye have been encapsulated into its oil core. These microspheres have previously been demonstrated to target cancer cells by functionalizing them with a layer of RGD peptides and could be functionalized with monoclonal antibodies. Preliminary results show that these magnetic microspheres, which are 2.05.0 microns in size, are readily detectable under MM-OCT when embedded in a 5\% agarose gel, in a 3-D scaffold of macrophage cells previously incubated with the microspheres, and when injected in-vivo into a tumor from an NMUcarcinogen rat animal model for breast cancer.
\end{abstract}

Keywords: Optical Coherence Tomography, Microspheres, Contrast Agent, Atherosclerosis, Cancer

\section{INTRODUCTION}

\subsection{Clinical Motivation}

Breast cancer is the second leading cause of cancer deaths in women and remains the most widely diagnosed type of cancer in women. There continues to be an increasing need for an imaging technology that can provide a real time microscopic assessment regarding the presence of cancer cells and perhaps even possibly stage the progression of the cancer. Optical coherence tomography (OCT) is capable of providing real-time 3-D structural images of the breast tissue with penetration depths of 1-2 mm. Currently, the staging of breast cancer is based on three main criteria: the size of the primary tumor, the infiltration of lymph nodes by cancer cells, and the subsequent metastasis of cancer cells to other sites. The status of lymph nodes has long been used by the medical community in helping stage the metastatic state of the cancer. In an MNU carcinogen induced rat animal model, the morphology of lymph nodes have been successfully imaged under structural OCT (1). However, the challenge still lies in the ability to detect and identify smaller and smaller nests of cancer cells or micro-metastases within these lymph nodes. The need to increase the molecular and cellular sensitivity can be addressed through the development of targeted contrast agents. By complimenting OCT's strength in providing 3-D volumetric structural information, targeted contrast agents would be able to further visualize and highlight cells and molecular markers of interest under OCT. By targeting the agents to overexpressed receptor sites in cancer cells and in the neovasculature such as the integrin receptors, cancer cells would become more readily apparent under structural OCT and its functional modalities. By complimenting the morphological information acquired through

Molecular Probes for Biomedical Applications II,

edited by Samuel Achilefu, Darryl J. Bornhop, Ramesh Raghavachari

Proc. of SPIE Vol. 6867, 68670F, (2008) - 1605-7422/08/\$18 - doi: 10.1117/12.762428

Proc. of SPIE Vol. $686768670 \mathrm{~F}-1$ 
structural OCT, contrast agents will be able to enhance the cellular and molecular sensitivity furthering the field of OCT in breast cancer.

However, the overexpression of integrin receptors is not unique to the development of cancer and angiogensis. There are some signs that integrin receptors play a role in the development of atherosclerosis and cardiovascular diseases which also affects a significant portion of the population. Atherosclerosis currently accounts for over $75 \%$ of the cardiovascular diseases including strokes (2). Strokes make up for approximately $18 \%$ of cardiovascular diseases according to the most current Heart Disease and Stroke Statistics from the AHA (2). As one of the leading causes of death and of long-term, severe disability, being able to identify the location and progression of atherosclerotic lesions has become a critical issue (2). Earlier detection and thereby the treatment to slow or eliminate the threat from atherosclerotic lesions is highly needed as most are not detected until an individual has experienced a heart attack or stroke. By developing an imaging technology that can provide real-time in-vivo information regarding the presence and perhaps even the earlier staging of atherosclerotic lesions would prove to be invaluable. Intravascular OCT is already proven to be more widely accepted and can already aid in the evaluation of the vasculature for the development of atherosclerotic lesions after they have been localized by other conventional methods such as CT, ultrasound, or fluoroscopy. OCT can provide real-time 3-D structural images of the cardiovasculature with penetration depths of 1-2 $\mathrm{mm}$. However, with the use of targeted contrast agents, it would be possible for atherosclerotic lesions and vulnerable plaques to be identified earlier.

The most promising and most traditional method of targeting atherosclerotic lesions and cancer is by examining the signaling pathways that are overexpressed such as the integrin receptors (alpha (v) beta (3), alpha (4) beta (1)), the growth factors (vascular endothelial growth factors, basic fibroblast growth factors), and the adhesion molecules (vascular cellular adhesion molecules, intercellular adhesion molecules). Here we are focused mostly on the integrin receptors which would be targeted using a fairly widely known and accepted use of the RGD peptide motif. Both diseases do overexpress the integrin receptors at these sites indicating a potential venue in the development of targeted OCT contrast agents.

\subsection{Optical Coherence Tomography}

OCT (3) has been extensively studied over the past decade in the field of cardiology (4-20). In comparison to intravascular ultrasound, OCT is able to provide much higher resolution by at least an order of magnitude (7-9). In addition, multiple OCT probe designs have been developed to fit inside intravascular catheters providing forward imaging OCT images and radial OCT images $(6,21)$. With increasing data acquisition and processing rates, studies have been shifted towards 3-D OCT sampling (22) which can be used as the probe is being fed through the vasculature or using an automatic pull back method to image key structures such as the atrioventricular node or to monitor the status of diseases such as atherosclerosis. However, the major drawback to the use of OCT in this particular medical field is the presence of blood. Red blood cells are highly scattering thereby largely attenuating the OCT signal making it harder to image via OCT unless a saline flush is performed prior to imaging to temporarily clear the area of blood. Despite this one drawback, OCT has been successfully used in the visualization of the vasculature, the use of stents, and most relevantly in the characterization of atherosclerosis $(4,8,11,12,15,17-19)$. In the case of stents, OCT imaging was performed prior to the deployment of the balloon and after the installation of the stents giving the surgeons an instantaneous assessment of the status of the stent and its role in re-expanding the vascular walls $(10,16)$. Using OCT, efforts have been made to quantify the activated macrophage content as well as to differentiate between the types of plaques found in the lesion (fibrous, fibrocalcific, or lipid-rich). Optical Doppler tomography (ODT) analogous to the Doppler ultrasound is capable of measuring blood flow.

Within the field of OCT, there has been a recent push to develop techniques to improve the molecular and cellular sensitivity of OCT (23-34). One such example has been pump-probe OCT which makes use of a pump-probe source to probe the ground state absorption of the molecule (27). Within our laboratories, we have been able to develop several techniques including Nonlinear Interferometric Vibrational Imaging (NIVI) to probe the molecular vibrations of the sample using an interferometer setup $(29,34)$. A second novel method is a functional OCT modality called magnetomotive OCT (MM-OCT) which makes use of an external magnetic field to magnetically modulate its corresponding contrast agent to induce the displacement of nearby scatterers $(32,33)$. Spectroscopic OCT (S-OCT), which is being researched by multiple groups, has shown some promising success in being able to provide quantitative information about the biochemical makeup or the presence of NIR dyes in the specimen as well as in the concentration of hemoglobin $(28,35,36)$. Polarization-sensitive OCT (PS-OCT) is another functional mode of OCT which measures the birefringence of the tissue (24). Birefringence refers to the change in the polarization of the back-scattered light due 
to the specimen. Changes in the ordered structure such as the actin filaments in muscle tissue or of collagen in skin has been thought to be attributed to the birefringence signal which can be measured using polarization-sensitive OCT. These techniques have focused on the detection of both endogeneous and exogenous particles. However, to increase the viability of OCT in detecting earlier changes in disease progression, the focus lies in the functional modalities of OCT where the tissue can be probed on a cellular and molecular level.

A functional OCT modality which will be the main focus of this report is MM-OCT (32) recently developed in our laboratories. The basic concept is to take the traditional OCT system and add a means to modulate an external magnetic field while doing the OCT imaging. The magnetic field is optimally modulated at half the axial scan frequency which consequently modulates the OCT image between an "on" and "off" state. By subtracting one signal from the other, one is able to generate an additional $30 \mathrm{~dB}$ in contrast to the traditional OCT image with the addition of magneticallysensitive contrast agents. A solenoid coil is placed on the distal end of the probing arm to create the gradient magnetic field close to the sample. Contrast agents with a magnetic susceptibility placed near the probe can now be controlled by an external magnetic field allowing them to be detected under MM-OCT. These nanoparticles themselves do not necessarily need to express significant scattering or absorbing properties in their optical profiles in order to be detected in MM-OCT, but simply need to be able to displace the tissue scatterers around them. This added dimensionality to the contrast matrix gives us another means to develop contrast agents for OCT.

\subsection{Protein Microsphere}

The contrast agent presented here is a modified protein microsphere $(23,26)$. These microspheres consist of a hydrophilic protein BSA (bovine serum albumin) shell and a hydrophobic vegetable oil core. Using high-frequency ultrasound, he sonication process creates a fluid mixing state allowing the protein to form a shell encapsulating the oil and hydrophobic nanoparticles. Traditionally these microspheres have been filled with air, perfluorocarbon, or oil in order to be used for ultrasound and provide a sound echo. However, the introduction of vegetable oil as the solvent of the inner core has resulted in a much more stable and longer lasting contrast agent extending its lifetime from three days to several months. The surfactant used to emulsify the microspheres is one of the main controlling factors in the size determination of the protein microspheres since it affects the surface tension between the oil and protein. The size of these microspheres can range anywhere from $200 \mathrm{~nm}$ to $15 \mu \mathrm{m}$ in diameter. Other factors that contribute to the physical properties of the protein microspheres include the sonication power, frequency, and the surfactant used.

In previously reported studies, these protein microspheres have been extensively modified to provide greater contrast for OCT by incorporating scattering and magnetic nanoparticles. Some earlier experiments have shown that one can change the effective refractive index of the modified protein microspheres by altering the shell/surface with certain nanoparticles whose refractive indices range from 0.18 (gold) to 3.08 (carbon) compared to the refractive index of tissue of 1.48. This change in refractive index highly contributes to the increased backscattering of the light and thus the OCT signal in the sample. These same microspheres have also been modified with iron oxide (magnetite) in the inner core for MM-OCT, presented here, and with near-infrared dyes for spectroscopic OCT. Iron oxide nanoparticles has been reported to provide a large contrast (up to $30 \mathrm{db}$ ) via the displacement of adjacent scatterers under MM-OCT. A similar phenomenon is expected to be seen once the iron-oxide nanoparticles are encapsulated within the protein microsphere. As an OCT contrast agent, the iron-oxide filled protein microspheres have been tested in a number of samples including layered tissue phantoms, in mouse organs, and in tadpoles. As expected, an added contrast is seen wherever the ironoxide had been deposited or uptaken by the cell, tissue, or animal.

\section{MATERIALS \& METHODS}

\subsection{Instrument Setup for Protein Microsphere Synthesis}

For the synthesis of protein microspheres with an optimum size range between 2-5 $\mu \mathrm{m}$, the ultrasound horn was operated at a frequency of $20 \mathrm{KHz}$ with an acoustic power of $35 \mathrm{Watts} / \mathrm{cm}^{2}$. A change in the acoustic power and ultrasound frequency will change the size distribution of the resulting microspheres. A change in the effective surface tension between the oil and water phases can also affect the size distribution of the synthesized microspheres as the surface tension will surely affect the cross-linking capability of the proteins in the shell.

Proc. of SPIE Vol. $686768670 \mathrm{~F}-3$ 


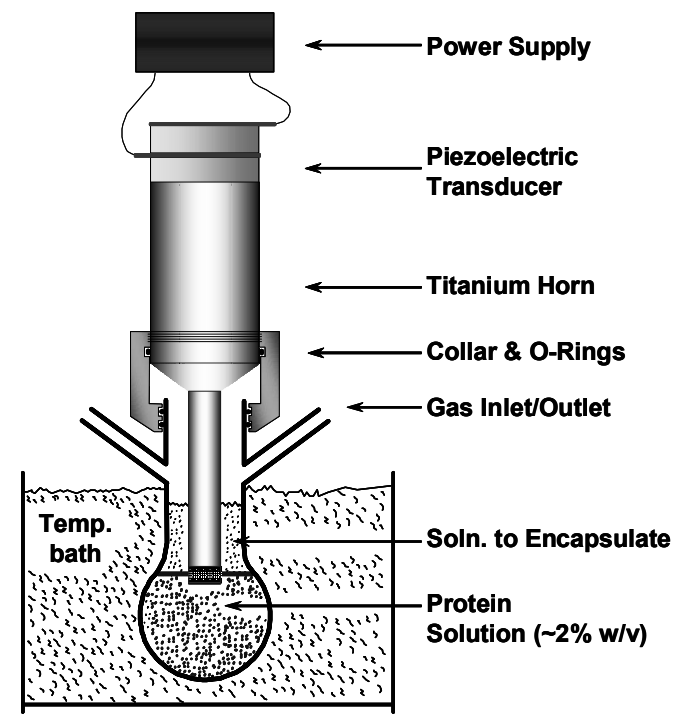

Fig. 1. The ultrasound titanium horn is placed in an upright configuration allowing a glass cell containing the oil layer (top layer) and the protein layer (bottom layer) to be attached firmly to the horn. The tip of the horn is optimally placed at the oil / water interface.

\subsection{Protein Microsphere Synthesis}

The synthesis of protein microspheres depends on three main concepts: the emulsification process, the aggregation of the protein at the oil / water interface, and the chemical crosslinking of the protein. In the case of BSA, the crosslinking occurs via the formed disulfide bonds through the inter-protein cysteine oxidation. The target modified protein microsphere synthesized in these studies contain an oil-filled core with $\mathrm{Fe}_{3} \mathrm{O}_{4}$ (iron oxide - magnetite) and Nile Red fluorescent dye and a BSA protein shell. $250 \mathrm{mg}$ of $\mathrm{Fe}_{3} \mathrm{O}_{4}$ and $1.5 \mathrm{mg}$ of Nile Red was dissolved into a $1 \mathrm{~mL}$ of hexane and sonicated for a period of five minutes and subsequently added to $10 \mathrm{~mL}$ of vegetable oil. The oil solution was sonicated for an additional five minutes and placed in a water bath at $80^{\circ} \mathrm{C}$ to allow the hexane to evaporate as the nanoparticles dissolved into the vegetable oil. Other particles that have been successfully encapsulated into the hydrophobic core include fluorescent dyes (Bodipy and Nile Red) and nanoparticles (iron oxide, melanin, carbon) (23).

The outer shell is the second component which can be altered in the protein microsphere. This is achieved by pre-mixing the nanoparticles of interest with the BSA protein similarly to when the nanoparticles were premixed with the oil to encapsulate the nanoparticles into the inner core. Prior to the mixing with the oil and sonication process, the protein is allowed bind to the nanoparticle via hydrophilic interactions. Currently, the outer protein shell measures approximately $50 \mathrm{~nm}$ in thickness. Different nanoparticles can be incorporated within this outer shell similarly to the inner core of the microsphere. For the microspheres used in these studies, no particles were introduced into the outer protein shell. Once these solutions have been individually mixed, the vegetable oil solution is layered on top of the $5 \%$ BSA protein solution inside sonochemical cell in a 30/70 ratio, respectively. The ultrasound horn is then lowered to the oil / water interface. The solution was emulsified using an ultrasound horn at a frequency of $20 \mathrm{KHz}$, and an acoustic power of $35 \mathrm{Watts} / \mathrm{cm}^{2}$ for a period of three minutes. During this time, it is critical to place the cell in a water bath at a temperature of $45^{\circ} \mathrm{C}$ to keep the BSA protein from denaturing. Once the microspheres have been synthesized, they were repeatedly washed nanopure water and a cold PBS buffer solution to remove any excess reagents. Using a size filter, the microspheres under $5 \mu \mathrm{m}$ were selected. Under these conditions, the size distribution of microspheres ranged between $2-5 \mu \mathrm{m}$.

\subsection{Targeting the Protein Microsphere: Layer-By-Layer Adhesion}

The integrin receptors, which are heterodimer, transmembrane receptors, have been widely reported as key players in angiogenesis and the proliferation of cancer metastases. With over 25 known integrin receptors, the one that has been targeted for this study is the alpha (v) beta (3) integrin receptor. The arginine-glycine-aspartate (RGD) sequence motif was initially used as a label to target tumor cells and atherosclerotic plaques. The alpha (v) beta (3) integrin receptor has been known to be highly overexpressed during the development of angiogenesis and tumor vasculature. However, it is also quite known as well that the use of RGD broadly targets integrin receptors and are not very specific to the alpha (v) 
beta (3) receptor. For these initial studies, the RGD peptide was embedded into a longer and highly positively charged polylysine sequence yielding the following sequence KKKKKKKRGD. This sequence was synthesized by the Protein Sciences Facility, Proteomics, Biotechnology Center at the University of Illinois at Urbana-Champaign.

Using layer-by-layer (LBL) adhesion, one can further modify the surface of the protein microspheres. This technique was used to functionalize the outer surface of the protein microsphere with RGD peptide sequences. Previous experiments have used this method to functionalize the outside of microspheres with various nanoparticles such as gold, quantum dots, iron oxide, and DNA to name a few $(23,26)$. However, in this study, we took advantage of this technique to add targeting agents to the outer surface of the protein microsphere. Using this method, the outer BSA protein shell was initially layered with PDDA and by being placed on a rotisserie shaker at $0^{\circ} \mathrm{C}$ for a period of 30 minutes. The targeting agent of interest for the integrin receptors in this study is the RGD peptide sequence. This positively charged sequence (KKKKKKKRGD) is the best sequence in terms of noncovalently binding the RGD peptide to the surface of the negatively charged protein microspheres using this Layer-By-Layer (LBL) approach. To continue with the LBL technique, the protein microspheres were resuspended in a solution of RGD peptides and placed on the rotisserie shaker for a period of 30 minutes at $0^{\circ} \mathrm{C}$. The adhesion of the RGD sequence was previously confirmed in other studies by layering a layer of silica nanoparticles which were negatively charged (37).

\subsection{Magnetomotive Optical Coherence Tomography (MM-OCT) Instrument}

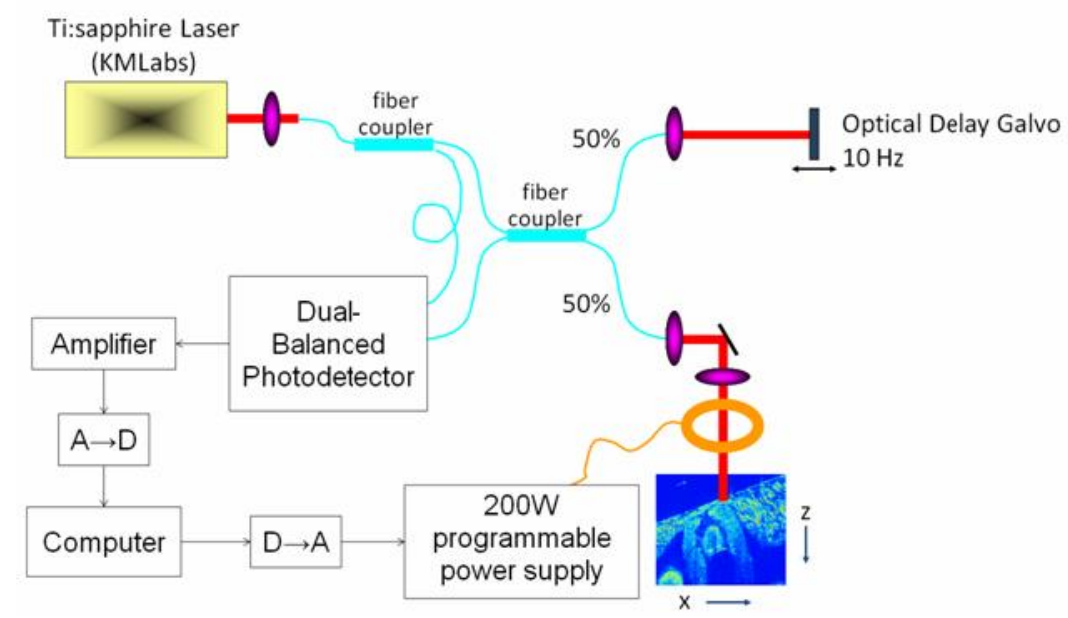

Fig. 2. Schematic of a time domain magnetomotive OCT (MM-OCT) system centered at $800 \mathrm{~nm}$ with a $100 \mathrm{~nm}$ bandwidth. This system provides an axial resolution of $3 \mu \mathrm{m}$ and a transverse resolution of $16 \mu \mathrm{m}$.

Here we illustrate a novel contrast agent technology designed for OCT, called magnetomotive OCT (MM-OCT) (32). Magnetically-responsive particles are used as passive or targeted contrast. An external magnetic field is applied to the local region where OCT signals are being acquired using a small solenoid coil generating a gradient field of 0.09T, with the imaging beam passing through the bore of the coil. By altering the applied magnetic field through a series of pulse sequences, the magnetic particles respond by displacing nearby scatterers in the surrounding cellular microenvironment. This change in the scattering profile can detected through lock-in detection using MM-OCT allowing us to functionally probe tissue with targeted magnetic particles. The MM-OCT system used in the following experiments were based on a time domain OCT system which had a center wavelength of $800 \mathrm{~nm}$ and a $100 \mathrm{~nm}$ bandwidth resulting in an axial resolution of $3 \mu \mathrm{m}$. The $40 \mathrm{~mm}$ focal length lens placed in the sample arm yielded a transverse resolution of $16 \mu \mathrm{m}$.

\subsection{5\% Agarose Gel Matrix}

To test the viability of these magnetic particles under MM-OCT, the magnetic protein microspheres were initially embedded into a 5\% Agarose gel matrix. These phantoms were made to provide a three-dimensional construct of the microspheres that closely mimics the mechanical properties of tissue. These experiments allowed us to study the viability of these microspheres in generating a detectable MM-OCT signal. The gel was allowed to solidify and subsequently imaged under MM-OCT. 


\subsection{Macrophage Scaffold}

To test the viability of the microspheres in a living environment, the microspheres were incubated with macrophages. The macrophages were incubated in cell media containing $10 \%$ iron oxide encapsulated protein microspheres for a period of four hours allowing sufficient time for the macrophages to find and to engulf the microspheres. The cells were then thoroughly washed with PBS buffer to remove any loose or excess microspheres. The cells were isolated and embedded into a 5\% Agarose Gel Matrix. The gel matrix did contain cell media to allow the macrophages to continue to survive after being embedded and during the imaging sessions. The gel was allowed to solidify and was imaged under MM-OCT.

\subsection{In-Vivo: NMU Carcinogen Induced Rat Mammary Model}

From previous OCT experiments, it has been well characterized that tumors were highly scattering and could potentially mask the magnetic signal of the microspheres. To assess the ability to detect these microspheres in highly scattering environments and to determine the effect of motion artifacts induced by the animal's breathing on the measured magnetic signal; these experiments were carried out in-vivo in an NMU carcinogen induced rat mammary model. The female Rat Wister-Furth rats were administered N-methyl-N-nitrosourea (NMU) dissolved in saline $(12.5 \mathrm{mg} / \mathrm{ml})$ and administered i.p. at $55 \mathrm{mg} / \mathrm{kg}$ body weight at 28 and 35 days of age. The injection volumes ranged from $0.2-0.4 \mathrm{ml}$ depending on the weight of the animal.

Seven days after the second injection, tumors were first palpable. The rats were identified for the study at the time when their tumors grew to a size of 1.0-1.5 $\mathrm{cm}$. Under anesthesia condition, the mammary tumors and surrounding area of the animal was exposed and imaged to provide a control image. At the site of the tumor, the contrast agent was directly injected into the tumor mass and imaged over a period of $1 \mathrm{hr}$ using OCT and MM-OCT. After imaging, all animals were euthanized by cervical dislocation while under anesthesia and confirmed by thoracotomy. The tumor masses were resected, fixed in formalin, sectioned, and H\&E stained.

\subsection{In-Vitro: HT-29 \& CRL-4010}

To test the targeting and binding specificity of the RGD coated protein microspheres, two different cell lines with different levels (normal and overexpressed) of expression of the integrin receptors were incubated with the targeted and non-targeted contrast agents. The CRL-4010 cell line is a normal human breast epithelium cell line which was expected to have a normal expression of the integrin receptors. The HT-29 is a colon cancer cell line which should have an overexpression of the integrin receptors. These cell lines were cultured until they reached $90 \%$ confluency and were incubated in a similar fashion as the macrophages with cell media that had been pre-mixed with nile red encapsaluated protein microspheres for a period of $2 \mathrm{hrs}$ and $4 \mathrm{hrs}$. After incubation, the cells were thoroughly washed using PBS buffer to remove any excess or loose contrast agents. The cells were subsequently bathed in the PBS buffer and imaged using a fluorescence microscope to observe the localization of the Nile Red encapsulated microspheres within these cells.

\subsection{Ex-Vivo: Atherosclerotic Rat Animal Model}

To the test the targeting and binding of the RGD coated protein microsphere in a small animal model, an atherosclerotic rat model was used. The lesions have been cited to be restricted to the aortic root and are characterized by the presence of increased levels of cholesterol and macrophages. After prolonged feeding, the collection of cellular debris and collagen would be observed. The rats were housed under the supervision of the Animal Facilities at the Beckman Institute for Advanced Science and Technology at the University of Illinois at Urbana-Champaign. After ten weeks of being put on a high fat and high cholesterol diet, the atherosclerotic rats were euthanized by asphyxiation with $\mathrm{CO}_{2}$, and the aorta was extracted for OCT imaging. A control rat was also taken at the same age. The aortas were subsequently incubated at $37^{\circ} \mathrm{C}$ in cell media pre-mixed with the RGD coated magnetic protein microspheres for a period of $4 \mathrm{hrs}$. The aortas were washed thoroughly with PBS and placed in a saline solution to remove any loose or excess contrast agent. The entire aortas were imaged under the MM-OCT system as previously described.

\section{DATA \& RESULTS}

\subsection{Protein Microspheres as an scattering OCT contrast agent}

As has been previously reported, these protein microspheres have been previously studied as scattering OCT contrast agents by loading up the microspheres with various nanoparticles that would induce a large change in index of refraction 
in comparison to the nearby tissue. In a similar fashion, these iron oxide encapsulated protein microspheres were initially tested for the same purpose.

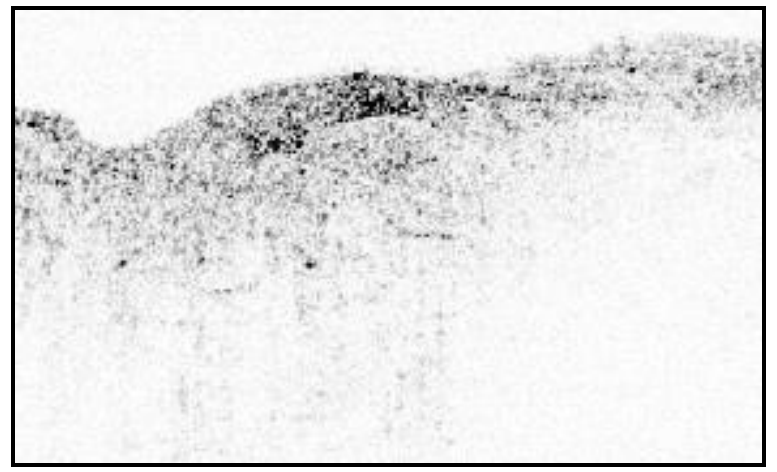

Fig. 3. OCT image using a time domain OCT system centered at $800 \mathrm{~nm}$ with a $100 \mathrm{~nm}$ bandwidth. This is an OCT image of a rat mammary tumor (in-vivo) that has been reflected and exposed. This image was taken prior to the injection of the rat with an RGD coated iron oxide encapsulated protein microsphere.

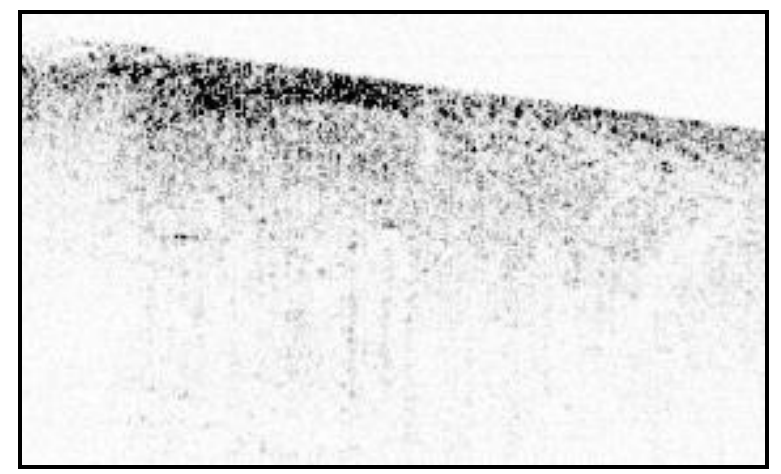

Fig. 4. OCT image using a time domain OCT system centered at $800 \mathrm{~nm}$ with a $100 \mathrm{~nm}$ bandwidth. This is an OCT image of a rat mammary tumor (in-vivo) that has been reflected and exposed. This image was taken 30 minutes after the injection of the rat with an RGD coated iron oxide encapsulated protein microsphere via the tail vein.

From the figures above, one can observe a slight increase in the scattering intensity in the area right above the tumor (middle of the image) which is where the tumor vasculature would be located. Since these microspheres are generally 2 $5 \mu \mathrm{m}$ in size, they are not expected to penetrate the vascular walls and enter into the tumor but instead to stay in the tumor vasculature. However, since this results proved to be inconclusive at the time, these iron oxide encapsulated protein microspheres were exploited for their magnetic properties.

\subsection{Protein Microspheres as an MM-OCT contrast agent}
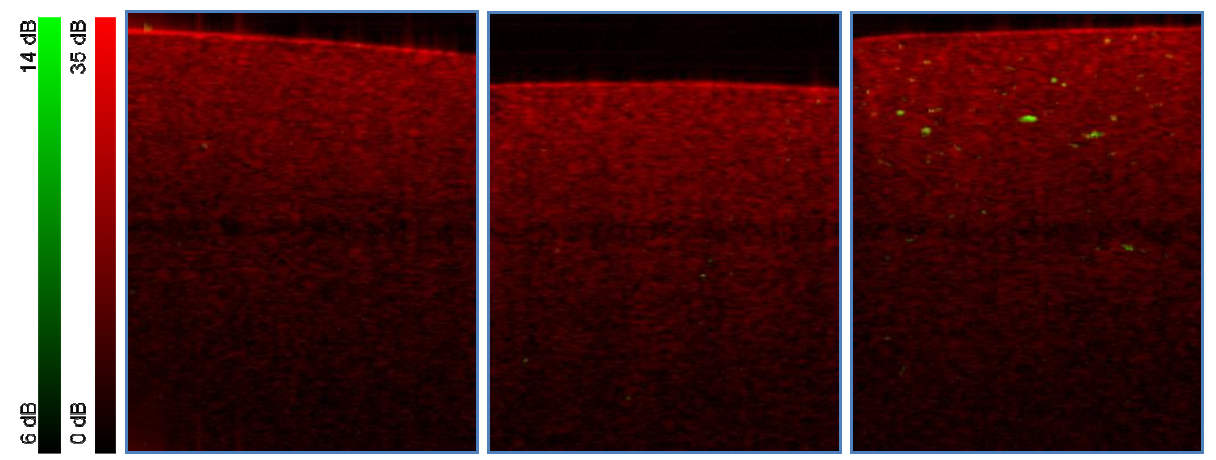

Fig. 5. Structural OCT image (red) using a time domain OCT system centered at $800 \mathrm{~nm}$ with a $100 \mathrm{~nm}$ bandwidth with the MM-OCT signal overlayed on top (green). This is an OCT image of three samples of 5\% Agarose gel that have been 
embedded with equal concentrations of plain microspheres (left), nile red encapsulated microspheres (middle), and nile red + iron oxide encapsulated microspheres (right) (images are $1 \mathrm{~mm}$ in width).

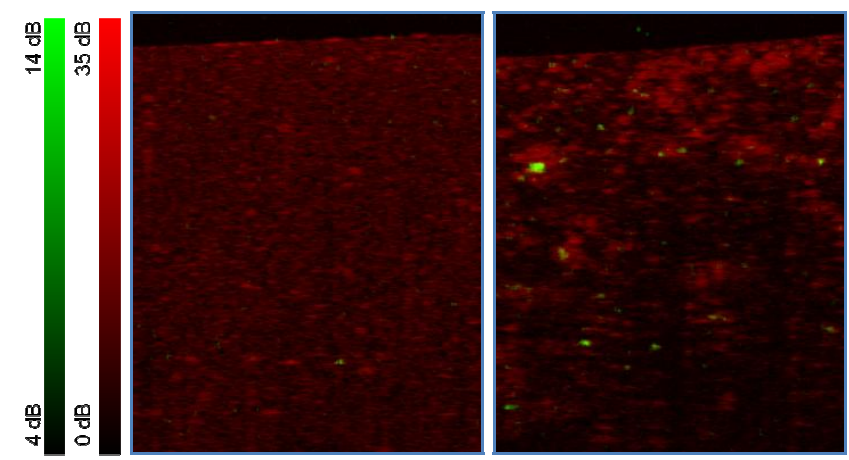

Fig. 6. Structural OCT image (red) using a time domain OCT system centered at $800 \mathrm{~nm}$ with a $100 \mathrm{~nm}$ bandwidth with the MM-OCT signal overlayed on top (green). This is an OCT image of two samples of macrophages embedded in 5\% Agarose gel. The macrophages can be seen in the structural OCT images as the larger scattering bodies. They were fed with equal concentrations of plain microspheres (left), and nile red + iron oxide encapsulated microspheres (right) (images are $1 \mathrm{~mm}$ in width).

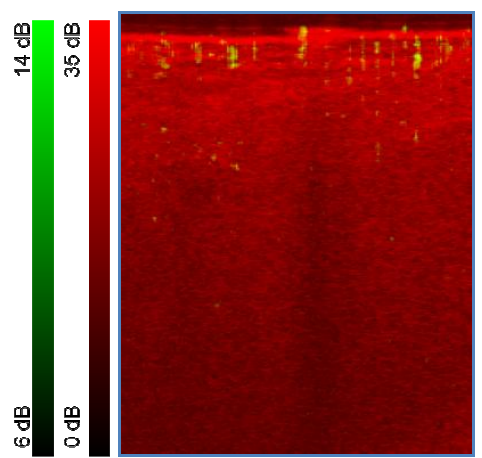

Fig. 7. Structural OCT image (red) using a time domain OCT system centered at $800 \mathrm{~nm}$ with a $100 \mathrm{~nm}$ bandwidth with the MM-OCT signal overlayed on top (green). This is an in-vivo OCT image of a rat mammary tumor that was injected with nile red + iron oxide encapsulated microspheres (image is $1 \mathrm{~mm}$ in width).

Based on Figure 5, one can clearly identify that the magnetic signal observed is coming from the presence of iron oxide inside of the protein microspheres and not from any other component of the modified protein microsphere. With Figure 6 , the magnetic signals are directly overlaid on top of some of the larger scattering bodies corresponding to the macrophages. This indicates that the macrophages have engulfed these particles and that iron oxide particles are localized to the interior of the cell after an incubation period of $4 \mathrm{hrs}$. Lastly based on the in-vivo tumor image in Figure 7 , one can observe that even in the highly scattering tumor tissue, the magnetic signal coming from the magnetic protein microspheres is still detectable mainly due to the fact we are able to externally modulate it.

Even the last experiment was done in-vivo, the time domain MM-OCT system was still capable of acquiring images accurately and rapidly enough such that the extraction of the magnetic signal was not affected by any incurred motion artifacts. In these images above, it is readily apparent that regardless of the environment in which these iron oxide encapsulated protein microspheres were embedded into such as Agarose gel, macrophages, or rat mammary tumors, the magnetic signal from these particles still proved to be strong enough to be detected under MM-OCT. 


\subsection{Targeting of Protein Microspheres to Integrin Receptors in Cancer}
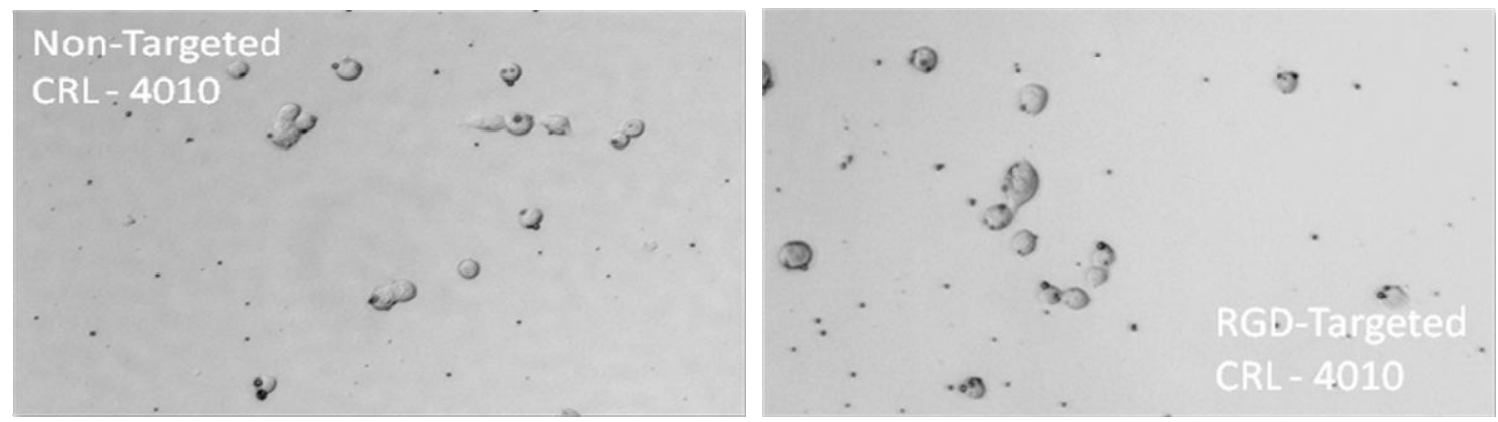

Fig. 8. Brightfield Images (10X) of CRL-4010 cells after they have been incubated for a period of 2 hrs with non-targeted (left) and targeted (right) nile red +iron oxide encapsulated protein microspheres.
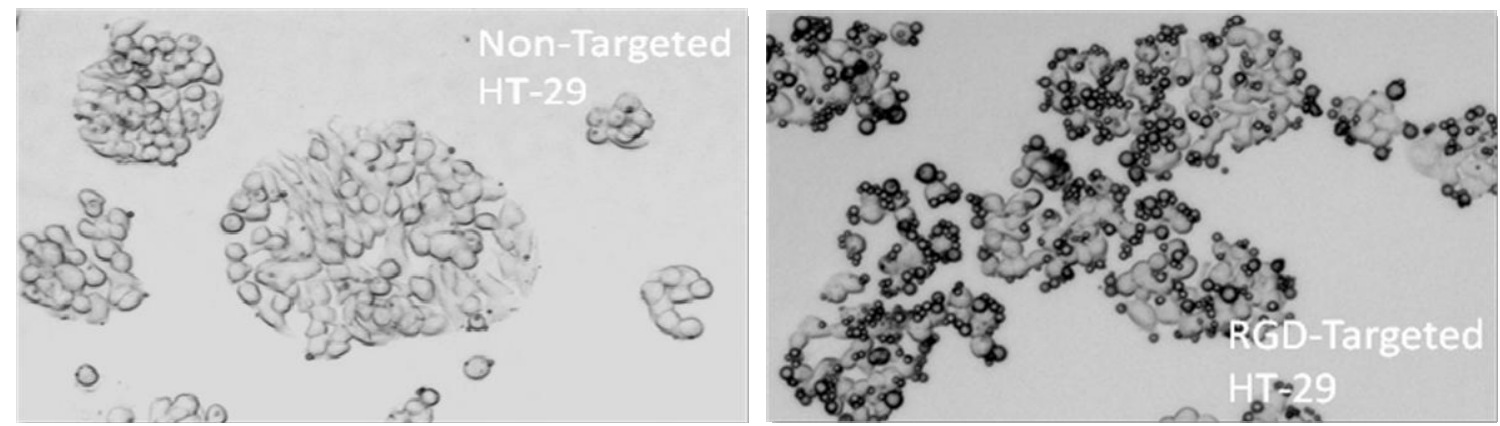

Fig. 9. Brightfield Images (10X) of HT-29 cells after they have been incubated for a period of 2 hrs with non-targeted (left) and targeted (right) nile red + iron oxide encapsulated protein microspheres.

These preliminary results clearly show the increased binding affinity of the targeted protein microspheres to the HT-29 cells with the increased expression of its integrin receptors as seen in the RGD Targeted HT-29 image. These images also indicate the high binding specificity for the HT-29 cells and not the CRL-4010 cells. Similarly, there is a low nonspecific binding (see the RGD Targeted CRL-4010) and low passive binding of the microspheres (see the non-targeted images).

\section{CONCLUSION}

In summary, we have been able to present a novel particle that is highly versatile in terms of its functionality and its potential as a contrast agent and in particular an MM-OCT contrast agent. The structure of these protein microspheres make them amenable for targeting the vascular walls using an RGD targeting motif and certainly biocompatible with their protein shell. The ability to encapsulate a wide array of magnetic particles or fluorescent dyes also makes it attractive as multimodal contrast agent with the potential to serve as a fluorescent label or an MRI contrast agent for more wide field, whole-body imaging. Once, these particles have been targeted and are localized to the region interest, a higher resolution imaging method such as OCT can be used to image the local environment on a micron scale. The ready uptake of these particles by cells such as the macrophages, or the in-vitro cell lines used in these studies also point to the potential use of these particles as drug delivery agents once they have been properly targeted.

\section{REFERENCES}

1. W. Luo, F. T. Nguyen, A. M. Zysk, T. S. Ralston, J. Brockenbrough, D. L. Marks, A. L. Oldenburg and S. A. Boppart, "Optical biopsy of lymph node morphology using optical coherence tomography," Technol Cancer Res Treat 4(5), 539-548 (2005)

2. "Heart Disease and Stroke Statistics — 2005 Update," American Heart Association, Dallas, Texas (2005). 
3. G. J. Tearney, M. E. Brezinski, B. E. Bouma, S. A. Boppart, C. Pitris, J. F. Southern and J. G. Fujimoto, "In vivo endoscopic optical biopsy with optical coherence tomography," Science 276(5321), 2037-2039 (1997)

4. M. E. Brezinski, G. J. Tearney, B. E. Bouma, S. A. Boppart, M. R. Hee, E. A. Swanson, J. F. Southern and J. G. Fujimoto, "Imaging of coronary artery microstructure (in vitro) with optical coherence tomography," Am J Cardiol 77(1), 92-93 (1996)

5. G. J. Tearney and B. E. Bouma, "Method and apparatus for determination of atherosclerotic plaque type by measurement of tissue optical properties," PCT Int. Appl. (2002)

6. G. J. Tearney, M. E. Brezinski, S. A. Boppart, B. E. Bouma, N. Weissman, J. F. Southern, E. A. Swanson and J. G. Fujimoto, "Images in cardiovascular medicine. Catheter-based optical imaging of a human coronary artery," Circulation 94(11), 3013 (1996)

7. M. E. Brezinski, G. J. Tearney, B. E. Bouma, J. A. Izatt, M. R. Hee, E. A. Swanson, J. F. Southern and J. G. Fujimoto, "Optical coherence tomography for optical biopsy. Properties and demonstration of vascular pathology," Circulation 93(6), 1206-1213 (1996)

8. M. E. Brezinski, G. J. Tearney, N. J. Weissman, S. A. Boppart, B. E. Bouma, M. R. Hee, A. E. Weyman, E. A. Swanson, J. F. Southern and J. G. Fujimoto, "Assessing atherosclerotic plaque morphology: comparison of optical coherence tomography and high frequency intravascular ultrasound," Heart 77(5), 397-403 (1997)

9. J. G. Fujimoto, S. A. Boppart, G. J. Tearney, B. E. Bouma, C. Pitris and M. E. Brezinski, "High resolution in vivo intra-arterial imaging with optical coherence tomography," Heart 82(2), 128-133 (1999)

10. I. K. Jang, G. Tearney and B. Bouma, "Visualization of tissue prolapse between coronary stent struts by optical coherence tomography: comparison with intravascular ultrasound," Circulation 104(22), 2754 (2001)

11. H. Yabushita, B. E. Bouma, S. L. Houser, H. T. Aretz, I. K. Jang, K. H. Schlendorf, C. R. Kauffman, M. Shishkov, D. H. Kang, E. F. Halpern and G. J. Tearney, "Characterization of human atherosclerosis by optical coherence tomography," Circulation 106(13), 1640-1645 (2002)

12. B. E. Bouma and G. J. Tearney, "Clinical imaging with optical coherence tomography," Acad Radiol 9(8), 942-953 (2002)

13. M. Gupta, A. M. Rollins, J. A. Izatt and I. R. Efimov, "Imaging of the atrioventricular node using optical coherence tomography," J Cardiovasc Electrophysiol 13(1), 95 (2002)

14. T. M. Yelbuz, M. A. Choma, L. Thrane, M. L. Kirby and J. A. Izatt, "Optical coherence tomography: a new highresolution imaging technology to study cardiac development in chick embryos," Circulation 106(22), 2771-2774 (2002)

15. I. K. Jang, B. E. Bouma, D. H. Kang, S. J. Park, S. W. Park, K. B. Seung, K. B. Choi, M. Shishkov, K. Schlendorf, E. Pomerantsev, S. L. Houser, H. T. Aretz and G. J. Tearney, "Visualization of coronary atherosclerotic plaques in patients using optical coherence tomography: comparison with intravascular ultrasound," J Am Coll Cardiol 39(4), 604-609 (2002)

16. B. E. Bouma, G. J. Tearney, H. Yabushita, M. Shishkov, C. R. Kauffman, D. DeJoseph Gauthier, B. D. MacNeill, S. L. Houser, H. T. Aretz, E. F. Halpern and I. K. Jang, "Evaluation of intracoronary stenting by intravascular optical coherence tomography," Heart 89(3), 317-320 (2003)

17. G. J. Tearney, H. Yabushita, S. L. Houser, H. T. Aretz, I. K. Jang, K. H. Schlendorf, C. R. Kauffman, M. Shishkov, E. F. Halpern and B. E. Bouma, "Quantification of macrophage content in atherosclerotic plaques by optical coherence tomography," Circulation 107(1), 113-119 (2003)

18. B. D. MacNeill, I. K. Jang, B. E. Bouma, N. Iftimia, M. Takano, H. Yabushita, M. Shishkov, C. R. Kauffman, S. L. Houser, H. T. Aretz, D. DeJoseph, E. F. Halpern and G. J. Tearney, "Focal and multi-focal plaque macrophage distributions in patients with acute and stable presentations of coronary artery disease," J Am Coll Cardiol 44(5), 972-979 (2004)

19. A. H. Chau, R. C. Chan, M. Shishkov, B. MacNeill, N. Iftimia, G. J. Tearney, R. D. Kamm, B. E. Bouma and M. R. Kaazempur-Mofrad, "Mechanical analysis of atherosclerotic plaques based on optical coherence tomography," Ann Biomed Eng 32(11), 1494-1503 (2004)

20. I. K. Jang, G. J. Tearney, B. MacNeill, M. Takano, F. Moselewski, N. Iftima, M. Shishkov, S. Houser, H. T. Aretz, E. F. Halpern and B. E. Bouma, "In vivo characterization of coronary atherosclerotic plaque by use of optical coherence tomography," Circulation 111(12), 1551-1555 (2005)

21. A. F. Zuluaga and B. E. Bouma, "Multi-channel optical coupler for spinning catheter," PCT Int. Appl. (2003)

22. D. Yelin, B. E. Bouma, N. Iftimia and G. J. Tearney, "Three-dimensional spectrally encoded imaging," Opt Lett 28(23), 2321-2323 (2003) 
23. T. M. Lee, A. L. Oldenburg, S. Sitafalwalla, D. L. Marks, W. Luo, F. J. Toublan, K. S. Suslick and S. A. Boppart, "Engineered microsphere contrast agents for optical coherence tomography," Opt Lett 28(17), 1546-1548 (2003)

24. J. E. Roth, J. A. Izatt and A. M. Rollins, "Method and apparatus for polarization-sensitive optical coherence tomography," PCT Int. Appl. (2002)

25. M. Lazebnik, D. L. Marks, K. Potgieter, R. Gillette and S. A. Boppart, "Functional optical coherence tomography for detecting neural activity through scattering changes," Opt Lett 28(14), 1218-1220 (2003)

26. F. Toublan, K. Suslick, S. Boppart, T. Lee and A. Oldenburg, "Modification of protein microspheres for biomedical application," Polymer Preprints 44(185 (2003)

27. K. D. Rao, M. A. Choma, S. Yazdanfar, A. M. Rollins and J. A. Izatt, "Molecular contrast in optical coherence tomography by use of a pump-probe technique," Opt Lett 28(5), 340-342 (2003)

28. C. Xu, J. Ye, D. L. Marks and S. A. Boppart, "Near-infrared dyes as contrast-enhancing agents for spectroscopic optical coherence tomography," Opt Lett 29(14), 1647-1649 (2004)

29. D. L. Marks and S. A. Boppart, "Nonlinear interferometric vibrational imaging," Phys Rev Lett 92(12), 123905 (2004)

30. C. Yang, M. A. Choma, L. E. Lamb, J. D. Simon and J. A. Izatt, "Protein-based molecular contrast optical coherence tomography with phytochrome as the contrast agent," Opt Lett 29(12), 1396-1398 (2004)

31. C. Yang, L. E. McGuckin, J. D. Simon, M. A. Choma, B. E. Applegate and J. A. Izatt, "Spectral triangulation molecular contrast optical coherence tomography with indocyanine green as the contrast agent," Opt Lett 29(17), 2016-2018 (2004)

32. A. L. Oldenburg, J. R. Gunther and S. A. Boppart, "Imaging magnetically labeled cells with magnetomotive optical coherence tomography," Opt Lett 30(7), 747-749 (2005)

33. C. Yang, "Molecular contrast optical coherence tomography: a review," Photochem Photobiol 81(2), 215-237 (2005)

34. J. S. Bredfeldt, C. Vinegoni, D. L. Marks and S. A. Boppart, "Molecularly sensitive optical coherence tomography," Opt Lett 30(5), 495-497 (2005)

35. T. Storen, A. Royset, L. O. Svaasand and T. Lindmo, "Functional imaging of dye concentration in tissue phantoms by spectroscopic optical coherence tomography," J Biomed Opt 10(2), 24037 (2005)

36. C. Xu, F. Kamalabadi and S. A. Boppart, "Comparative performance analysis of time-frequency distributions for spectroscopic optical coherence tomography," Appl Opt 44(10), 1813-1822 (2005)

37. F. J.-J. Toublan, "Methods to Tailor Protein Microspheres For Biomedical Applications," in Chemistry, University of Illinois at Urbana-Champaign, Urbana (2005). 